\title{
Improved Method for Direct Black- Box Arc Parameter Determination and Model Validation
}

\section{Journal Article}

Author(s):

Walter, Michael M.; Franck, Christian (1)

Publication date:

2014-04

Permanent link:

https://doi.org/10.3929/ethz-b-000082812

Rights / license:

In Copyright - Non-Commercial Use Permitted

Originally published in:

IEEE Transactions on Power Delivery 29(2), https://doi.org/10.1109/TPWRD.2013.2283278 


\section{(C) 2014 IEEE.}

Personal use of this material is permitted. Permission from IEEE must be obtained for all other uses, in any current or future media, including reprinting/republishing this material for advertising or promotional purposes, creating new collective works, for resale or redistribution to servers or lists, or reuse of any copyrighted component of this work in other works."

Digital Object Identifier: 10.1109/TPWRD.2013.2283278 


\title{
Improved method for direct black-box arc parameter determination and model validation
}

\author{
Michael M. Walter, Christian M. Franck, Senior Member, IEEE
}

\begin{abstract}
Black-box models are a valuable tool to simulate dynamic arc-network interactions, such as those occurring during fault current interruption in AC or HVDC circuit breakers. However, accurate determination of characteristic arc parameters from voltage and current measurements is very challenging. The paper aims to improve the accuracy in arc characterization. A new arbitrary current source is used to simplify arc parameter determination through a more complex current waveform, e. g. a series of staircase-like increasing steps. With this, the stationary and transient arc characteristics can be measured independently from each other. Therefore, current steps are well suited to a) validate the choice of a specific black box model and b) determine the arc parameters of the chosen model with higher accuracy. In principle, the source is also well suited to fill a table of the arc voltage response $U, \dot{U}$ at various current levels and slopes $I, \dot{I}$. Thus, it would be possible to characterize arcs independent of any black-box model, but it would not be well suited to compare different arcs. Therefore, the paper focuses on an improved blackbox parameter determination and the experimental validation of the Schwarz-model, with $P$ and $\tau$ being free functions of $g$.
\end{abstract}

Index Terms-arc discharges, high-voltage techniques, circuit breaker testing, HVDC circuit breakers, AC circuit breakers, HVDC interrupters, plasma.

\section{INTRODUCTION}

D ESPITE the large progress in physical arc modeling [1], [2], [3], [4], black-box models are still used to simulate dynamic arc-network interactions due to their low calculation effort [5], [6]. However, the validity and applicability of these models is often challenged due to multiple reasons. Firstly, their accuracy strongly depends on the exact description of the arc parameter functions which are very difficult to determine. Secondly, it is not straightforward to transfer a measured set of parameters to other arc conditions. This requires that the parameters are determined for each arc configuration. Moreover, there have been doubts that the arc may not be described with a single ordinary differential equation having exclusively the conductance $g$ as state variable [7].

Physical arc models often describe only the stationary arc behavior or, if dynamic behavior is included, they are applicable only for arcs under special conditions [8]. Apart from that they usually fail to model the dynamic behavior due to mathematical problems. However, in many applications the correct modeling of the high frequency interaction between the arc and its surrounding network is central. Passive resonance HVDC circuit breakers, for example, create an artificial current-zero crossing by exciting a high-frequency oscillation current between the arc-chamber and a parallel

M. M. Walter and C. M. Franck are with the Power Systems and High Voltage Laboratories, ETH Zurich, 8092 Zurich, Switzerland, (e-mail: michael.walter@alumni.ethz.ch; franck@eeh.ee.ethz.ch)

Manuscript submitted May, 2013.
LC-path [9], [10]. In this situation, dynamic arc-behavior occurs with high frequencies $(>5 \mathrm{kHz})$ and large current gradients $(>10 \mathrm{kA} / \mathrm{ms})$. Black-box models have already been successfully applied to describe and predict passive resonance arc behavior [9], [11].

The authors agree with the above concerns about blackbox models but believe that many of them originate from imprecise arc parameter determination by experiments and the inability to validate the chosen black-box modeling equation for the arc under investigation. The choice of a more complex black-box model is only possible if its parameters can still be determined experimentally. Classically, arcs are investigated using sinusoidal test currents which have coupled current amplitude $I$ and gradient $\dot{I}$. This current shape is not ideal for parameter evaluation, it would be preferred to control $I$ and $\dot{I}$ independently of each other.

The choice of black-box model equations and the algorithm to determine their parameters typically requires to make certain assumptions about the arc behavior. These assumptions often remain unverified when an arc is characterized experimentally. Firstly, one has to be aware, that a process including different physical processes is described by a simple first order differential equation, which may not describe the arc completely. In addition to that, some parameter determination methods introduce an analytical shape how the parameters depend on the arc state, e.g. an analytical expression of the arc cooling power $P$ as a function of the arc conductance $g$ : $P=P(g)$. The terminology direct and indirect are introduced to classify the methods. A determination method is classified as direct, if it does not require a mathematical description of the chosen arc parameters, but extracts them as individual measurement points at different arc states. Indirect methods, in contrast, require that the shape of the arc parameters is described, e.g. the often used power functions $P(g)=a \cdot g^{b}$.

In this contribution it is illustrated how a current source that is able to produce complex waveforms, can be used for an improved arc characterization. It can be used i) to characterize the arc by analyzing the step response to sudden changes in arc current before selecting a black-box model, ii) to judge which model equation is most suitable to describe the arc, and iii) to improve the accuracy of arc model parameter determination. This is achieved by decoupled creation of steady-state and dynamic arc behavior within one measurement and will be experimentally illustrated for a free burning arc.

The manuscript is structured as follows: sec. II briefly reviews classical direct and indirect methods for arc model parameter determination. In sec. III a new method based on staircase-like currents is proposed. This current shape is used to extract the parameters of a widely accepted black-box model 
and validate its applicability for the investigated arc. The classical and new methods are applied to the measurements of a free burning arc in sec. IV. The discussion of the results is made in sec. V. A summary and outlook concludes the paper in sec. VI.

\section{BLACK-BOX-MODELING OF ARCS}

The majority of today's black-box models are modifications or extensions of the well known models from Mayr and Cassie and can be brought into the form of eq. (1) [12], [13], [14], [15], [16]. This equation, also referred to as energy balance equation, describes the change of the stored energy content $Q$ in the arc column resulting from the imbalance between ohmic heating $P_{\text {heat }}$ and cooling $P_{\text {cool }}$ :

$$
\mathrm{d} Q / \mathrm{d} t=P_{\text {heat }}-P_{\text {cool }} .
$$

Under the assumption that the cooling power $P(Q)$ and the conductance $g(Q)$ are arbitrary functions of $Q$, the general form of the dynamic arc equation [16][17] can be formulated:

$$
\dot{g}=\frac{1}{\frac{\mathrm{d} Q}{\mathrm{~d} g}}\left(\frac{i^{2}}{g}-P\right)
$$

With the further assumption of $Q=Q_{0} \cdot \ln \left(g / G_{0}\right)$, where $Q_{0}$ and $G_{0}$ are constants describing the arc, plus the definition of a thermal time constant $\tau:=Q_{0} / P$, eq. (2) equates into the Mayr equation:

$$
\frac{\dot{g}}{g} \cdot P \tau=\frac{i^{2}}{g}-P .
$$

"Rejecting the hypothesis that in Mayr's model of a dynamic arc, represented by eq. 3, the time constant $\tau$ and cooling power $P$ are constants, leads to a generalized Mayr's equation or the Mayr-Schwarz arc equation" [17], where $P(g)$ and $\tau(g)$ are free functions of the arc conductance $g$

$$
\dot{g}=\frac{g}{\tau(g)}\left(\frac{u i}{P(g)}-1\right) \text {. }
$$

Generally, the determination of an arc characteristic is performed in two or three steps, in which the second step is optional:

1) Choice of a black-box equation to link the internally stored energy $Q$ to externally measurable parameters $u$ and $i$. This includes specification of arc parameters, which can be arbitrary function of the arc state, e.g. $P(g)$ and $\tau(g)$ [14].

2) Optionally, the chosen arc parameters are assumed to follow a specific analytic form, e.g. $P:=a \cdot g^{b}$ and $\tau:=c \cdot g^{d}[14]$. According to the classification above, determination methods that require such an expression would be called indirect.

3) A method is chosen to determine the specified arc model parameters of the chosen modeling-equation from measured $u$ and $i$ oscillograms. Thereby, direct determination requires a large measurement effort because the parameters must be measured and determined individually (with a reasonable discretization) for each possible arc state $g$. Indirect determination requires significantly less measurement and calculation effort because not the arc parameters but only their coefficients are determined, like $a, b, c, d$ in the example above.

The desire to characterize the arc independent of a blackbox model is to a certain point unrealistic, because it would require to tabulate the arc voltage and change of arc voltage for all current values and changes in current for all external arcing conditions. Even though this would be possible with our new current source, it would cause unnecessary large experimental effort and is not well suited to compare arcs under different external conditions. It is thus preferred to use the source to validate the applicability of a black-box model and to determine the arc model parameters directly and with an improved accuracy.

\section{A. Iterative indirect method (A)}

Several authors found experimentally over a wide range of conductance a potential dependency $P=a \cdot g^{b}$ and $\tau=c \cdot g^{d}$ [14], [18], [16]. This enables a numerical calculation of the arc voltage by integrating eq. (4) as a response to the measured current $i(t)$. Iteratively, the parameters are adjusted to minimize the least square error between the measured voltage $u(t)$ and recalculated waveform $u^{\star}(t)=f(i(t), a, b, c, d)$ [14].

\section{B. Direct parameter separation method (B)}

Rijanto [19] determines the parameters $P$ and $\tau$ only at specific instants in time $t=t_{0}$ with $i_{0}, u_{0}$ and $g_{0}=i_{0} / u_{0}$, in which the effect of either $P$ or $\tau$ disappears. In a local thermal equilibrium $\dot{g}_{0}=0$, the cooling power equals the ohmic heating power and eq. (4) simplifies to $P\left(g_{0}\right)=i_{0} \cdot u_{0}$. In a current zero crossing $i_{0}=0$, no ohmic heating is present and eq. (4) simplifies to $\tau=-g_{0} / \dot{g}_{0}$.

\section{Direct multiple gradient method $(C)$}

Amsinck [20], Ruppe [21], Drebenstedt [22] and Leu [11] transform eq. (4) either graphically or mathematically to the form of a straight line $y=m x+n$ with $m=P \tau$ and $n=P$.

$$
i \cdot u=(P \tau) \cdot(\dot{g} / g)+P .
$$

Eq. (5) is then solved at least for two points $x_{1}\left(u_{1}, i_{1}\right)$, $x_{2}\left(u_{2}, i_{2}\right)$ with identical conductance $g_{1}=i_{1} / u_{1}=g_{2}=i_{2} / u_{2}$ but different gradient $\dot{g}_{1} \neq \dot{g}_{2}$.

For only two points it equates to [20], [21]:

$$
\begin{gathered}
P=\frac{i_{1}^{2} \dot{g}_{2}-i_{2}^{2} \dot{g}_{1}}{g\left(\dot{g}_{2}-\dot{g}_{1}\right)} \\
\tau=\frac{g\left(i_{2}^{2}-i_{1}^{2}\right)}{i_{1}^{2} \dot{g}_{2}-i_{2}^{2} \dot{g}_{1}} .
\end{gathered}
$$

When using multiple points of identical conductance $g_{0}$, a least square fit can be applied to determine the straight line parameters $m$ and $n$ [22], [11]. The arc parameters equate from $P\left(g_{0}\right)=n$ and $\tau\left(g_{0}\right)=m / n$. 


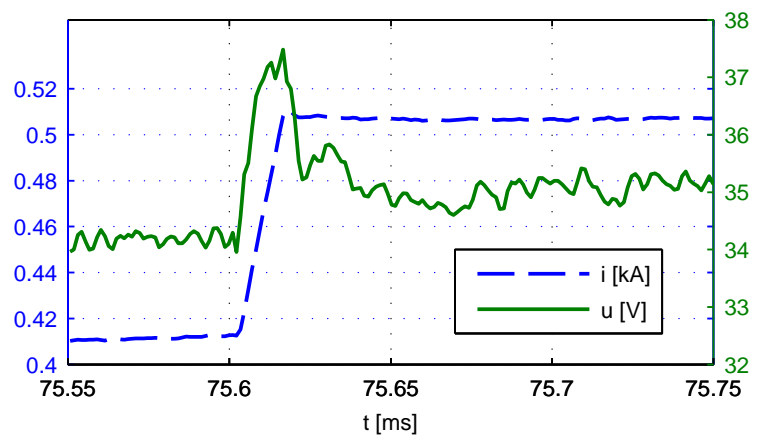

Fig. 1. Arc voltage response of a single current step.

\section{IMPROVED METHOD FOR DIRECT ARC CHARACTERIZATION}

\section{A. Arc characterization for choice of appropriate model}

A novel current source allows testing with nearly arbitrary current waveforms including steps and slopes of varying steepness [23]. Based on this ability, a new arc parameter determination method was developed that uses a series of staircase-like current steps for arc characterization. Herein, the current gradient is chosen as large as possible but at least sufficient to create a significant overshoot in the arc voltage. The subsequent constant current is chosen sufficiently long for the arc voltage to reach a stable voltage. The step amplitude must be chosen small enough that a linear shape of the stationary characteristic can be assumed between two successive constant current values.

The arc voltage response $u(t)$ of a single current step $i(t)$ is shown in Fig. 1. The arc shows a stationary characteristic during the constant current intervals, but fluctuations are superimposed. Further, a transient arc voltage spike occurs during the current step followed by a subsequent exponential decrease in the constant current phase towards the new stationary voltage.

From the observed transients one can conclude that the freeburning arc under investigations is quite well described by a first order differential equation, i.e. the current steps are used to identify a suitable arc model. In agreement with other authors equation (4) is thus chosen [14]. It is now required to determine the parameters $P(g)$ and $\tau(g)$ as free functions of g. An improved method for their determination is proposed in the following subsection.

Instead of introducing a black-box model at this point, the arc could be characterized by a table with external measurable parameters $U, \dot{U}$ as a response to several combinations of $I, \dot{I}$. To fill such a table, a large number of similar stairs would have to be measured with different current gradient $\dot{I}$ of the rising slope. We would consider such a description modelindependent, but very impractical to realize.

\section{B. Staircase method for improved and direct arc parameter determination (method D)}

It was found that current steps are also well suited to extract the arc parameters of a chosen model, as the stationary and transient arc characteristics are measured independently from each other. A similar approach has has been proposed in 1954 [7], but it was not experimentally realizable at this time.

The specific determination algorithm is shown here at the example of the generalized Mayr's equation (4). However, it is not restricted to this model and can be used more generally.

1) The measured voltage $u(t)$ and current $i(t)$ is cut into sections $u_{j}(t), i_{j}(t)$ each including a current step and the subsequent constant current.

2) In each section, the quasi stationary voltage $U_{j}$ and current $I_{j}$ after the dynamic behavior vanished are taken. The stationary cooling power follows directly as $P_{j}=I_{j} \cdot U_{j}$ versus conductance $G_{j}=I_{j} / U_{j}$.

3) $P_{j}\left(G_{j}\right)$ is section wise linearly interpolated between two quasi stationary points:

$$
p_{j}\left(g_{j}\right)=a_{j} * g+b_{j}
$$

with $a_{j}=\frac{P_{j}-P_{j-1}}{G_{j}-G_{j-1}}$ and $b_{j}=P_{j}-a_{j} * G_{j}$.

4) The conductance $g_{j}(t)=i_{j} / u_{j}$ and its gradient $\dot{g}_{j}(t)$ is calculated for each section. The chosen model equation (here equation 4) is also solved for the current gradient using equation 8 .

$$
{\dot{g_{j}}}^{*}(t)=\frac{1}{\tau_{j}}\left(\frac{i^{2}}{\left(a_{j} g_{j}+b_{j}\right)}-g_{j}\right)
$$

5) Iteratively for each of the segments $j$ the least square error

$$
\sum_{t}\left(\dot{g}_{j}-\dot{g}_{j}^{*}\right)^{2}
$$

is then minimized by modifying $\tau_{j}$ with a start guess, in our case, $\tau_{j} \approx 10 \mu$ s or $\tau_{j}=\tau_{j-1}$.

\section{EXPERIMENTAL SETUP AND METHOD IMPLEMENTATION}

\section{A. Arc}

The methods should be tested under worst case conditions concerning the signal to noise ratio between the currentgradient-excited transient over-voltage overshoot and the notcurrent-gradient-excited stochastic fluctuations. With respect to that, a free-burning horizontal arc is optimal because it features a relatively low voltage but strong fluctuations and thus has a poor signal to noise ratio. Experiments with wallstabilized arcs and blow arcs under stair currents have been performed and parameters curves were successfully extracted from them but they are not shown here. In comparison to free - burning arcs, these experiments had higher arc voltages and exhibit less arc fluctuations.

Spherical copper electrodes of $50 \mathrm{~mm}$ radius and $4 \mathrm{~mm}$ gap distance were used. The arc was ignited with a spark plug for the experiments in the LC-resonance test circuit. For the measurements in the new arbitrary current source, a copper igniting wire with $50 \mu \mathrm{m}$ diameter was used. To minimize the effect of the igniting wire on the arc characteristic, a constant current of $75 \mathrm{~A}$ was applied for at least $100 \mathrm{~ms}$ before the evaluated current section. 


\section{B. LC-resonance test current circuit}

An LC resonance circuit with pre-charged capacitor was used to generate the damped sinusoidal current. No additional damping resistor was added, but the current amplitude decrease resulted from the energy consumption of the arc and the resistance of the test circuit. Inductances and capacitances in the range of $4 \mu \mathrm{H} \ldots 10 \mathrm{mH}$ and $3.5 \ldots 14 \mu \mathrm{F}$ were used, respectively. The resulting oscillating frequency of the test current was in the range of $0.5 \ldots 17 \mathrm{kHz}$.

\section{Arbitrary current source}

Our newly built arbitrary current source was used to generate the desired staircase-like current waveform. The source consists of three interleaved buck-converter-type modules equipped with a real time IGBT controller. The current source concept has been published in [23] and will not be repeated in detail here.

\section{Current and voltage measurement}

A $1: 1000$ pearson current-transformer was used to measure the sinusoidal currents and an $A B B-E S 2000$ hall-sensor for the step currents. Both sensors have errors $<1 \%$. The arc voltage was measured from the signal difference of two identical LeCroy 1 : 1000 voltage probes with $<1 \%$ error, connected to a differential amplifier. Geometric constraints made it impossible to place the voltage probes directly at the electrode tips, which caused a stray inductance in series to the arc. The value of it was determined from a short circuit measurement to $L=0.45 \mu \mathrm{H}$. The voltage measurements were mathematically compensated for this stray inductance using the gradient of the measured current. The series resistance between the two voltage probes is negligible.

\section{E. Method implementation and validation}

The classical evaluation methods A-C and the improved method D, were implemented in Matlab. All implementations were carefully verified and validated using synthetic test data:

1) Validation: Hypothetical arc voltages $u_{h}(t)$ for various forms of assumed arc parameter functions $P_{h}(g)$ and $\tau_{h}(g)$ were calculated for current $i_{h}(t)$ with shapes similar to the measured ones. Then $P_{h}$ and $\tau_{h}$ were evaluated using methods A-D. Methods B-D always correctly recalculated the assumed parameter functions. Method A only succeeded for parameter shapes of the form $P_{h}=a \cdot g^{b}$ and $\tau_{h}=c \cdot g^{d}$.

2) Sensitivity to noise: Stochastic fluctuations in the arc voltage are always present in real measurements [24]. To determine the sensitivity of the different methods to noise, a white gaussian noise with vanishing mean value and standard deviation of $1 \mathrm{~V}$ was added to the hypothetical arc. This corresponds to $\approx 2 \%$ of the maximum arc voltage, which is considered to be a conservative lower limit. Again, a parameter evaluation was performed with methods A-D from the disturbed voltage signal. Both parameters $P_{h}$ and $\tau_{h}$ were correctly derived from methods $\mathrm{A}$ and $\mathrm{D}$ with minor error $(<1 \%)$. Methods B and C showed significant scattering $(>20 \%)$ in $P_{h}(g)$ and returned unreasonably high or even negative values of $\tau_{h}(g)$. Unlike methods A and D, methods B and $\mathrm{C}$ use single points in time to calculate the arc parameters. Therefore, they are much more sensitive in disturbances in measurement values.

3) Choice of filtering: It was tried to improve the $\tau$ determination from methods B and C by numerically filtering $u_{h}(t)$ before. A $5^{\text {th }}$ order butterworth filter with cut-off frequency at $100 \times$ the current oscillation frequency was able to reduce errors and scattering of method B below $10 \%$. For method C, the filter reduced the error in $P_{h}$ below $10 \%$, but the error in $\tau_{j}\left(g_{j}\right)$ remained above $50 \%$. Reason for the poor $\tau$ evaluation of method $\mathrm{C}$ lies in the error amplification through the straight line fit.

\section{Results}

The improved method for arc parameter determination should be compared with the existing methods. The existing methods are all based on the generalized Mayr's equation. Therefore, the comparison is performed for its parameter functions $P(g)$ and $\tau(g)$. As verified above, eq. (4) characterizes the arc under investigation sufficiently accurate.

\section{A. Classical methods A-C}

1) Measurement results: Sinusoidal currents of 13 frequencies in the range of $0.5 \ldots 16 \mathrm{kHz}$ were created and applied to the arc as described in section IV-A. Of these, only three test currents and the corresponding voltages are shown in Figs. 2a)c). The voltage waveforms show re-ignition peaks shortly after current zero only for low current oscillation amplitude and for low frequency oscillations. Only those conditions show sufficiently small gradients at current zero so that the arc reduces its conductance significantly. For increasing frequencies, the measurement gets closer to a sinusoidal arc voltage that is in phase with the current. For infinite frequency, the arc would show purely resistive behavior.

2) Parameter evaluation results: Methods B and C were applied separately to each measurement to extract the parameters $P(g)$ and $\tau(g)$. Method A was applied to all selected measurements in Figs. 2a-c) simultaneously. The resulting $P(g)$ are shown for three test current frequencies in Figs. 2d)f) and the resulting $\tau(g)$ in Figs. $2 \mathrm{~g})-\mathrm{i}$ ).

Method A yields a linearly rising $P=a \cdot g^{b}$ with $a=$ $1560 \mathrm{~W} / \mathrm{s}$ and $b=1$. Methods $\mathrm{B}$ and $\mathrm{C}$ extracted almost the same linearly rising power loss for all frequencies. Methods B and $\mathrm{C}$ show an increasing scattering with increasing frequency. In method $\mathrm{B}$ this is caused by error propagation. The time the arc remains at $\dot{g} \approx 0$ is smaller for higher frequencies and thus present scattering in measurement voltage and current can be averaged out less. For method $\mathrm{C}$, the reason lies in the arc behavior itself. At low frequencies, the arc follows mainly its stationary curve. Consequently, lower current gradients, i.e. lower oscillation frequencies, lead to more accurate $P(g)$ results.

Methods A-C all extracted arc thermal inertias of $0-20 \mu \mathrm{s}$. However, the resulting $\tau$ strongly varies with method and current oscillation frequency: Method A results in a curve $\tau(g)=c \cdot g^{d}$ with $c=4.3 \mu$ s and $d=0.3$. A similar result 

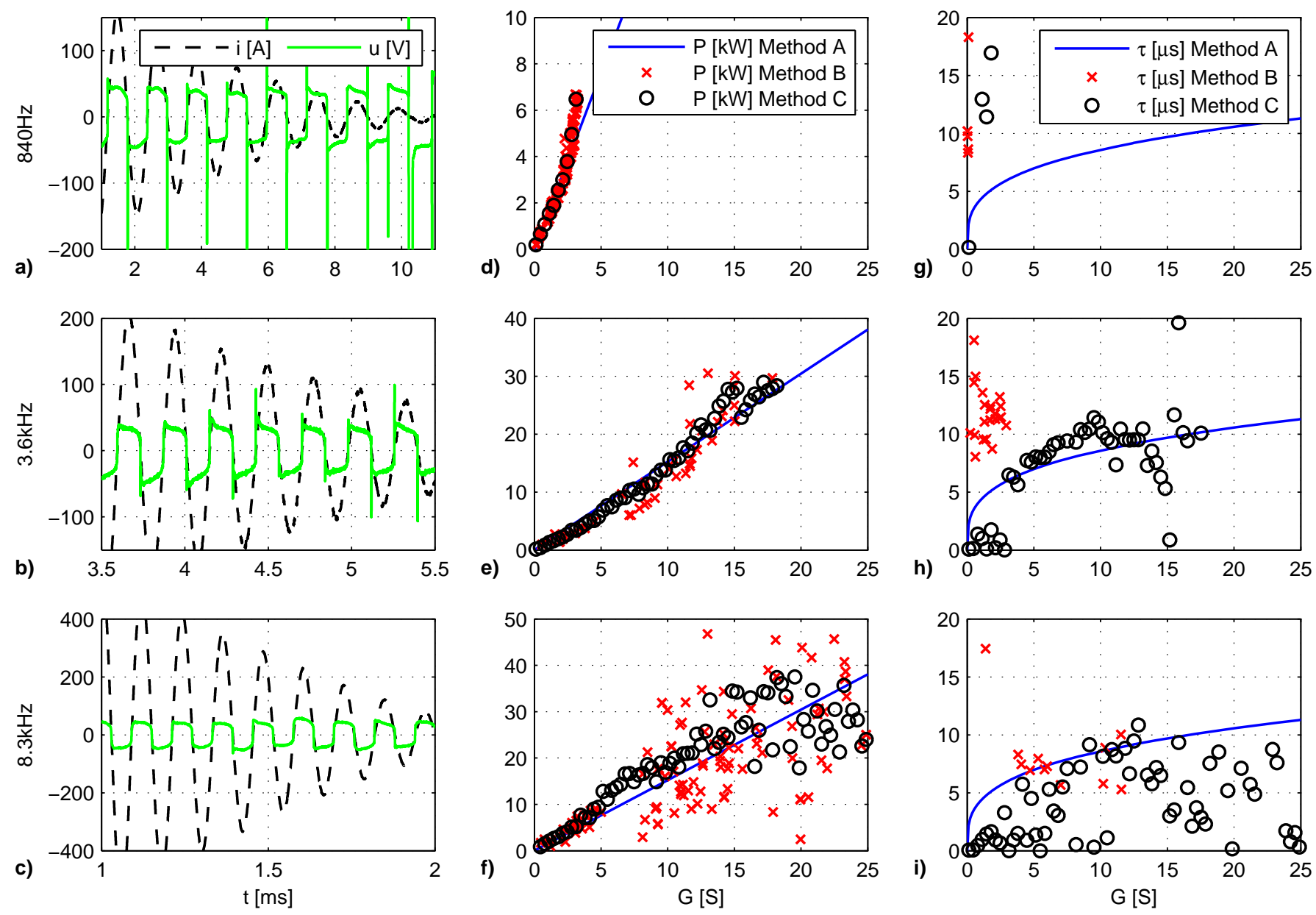

Fig. 2. Experimental results of LC-currents (classical methods): a)-c) current and voltage waveforms, d)-f) arc power loss $P(g)$, g)-i) thermal inertia $\tau(g)$.

was found for the $8.4 \mathrm{kHz}$ measurement with method B and for the $3.5 \mathrm{kHz}$ measurement with method $\mathrm{C}$.

For a reliable evaluation also at high conductance values, method B requires high frequency oscillations as it uses current zero crossings for the $\tau$ evaluation. At low conductances, method B yielded unexpectedly high $\tau$ values.

Method $\mathrm{C}$ extracts $P$ and $\tau$ simultaneously and, consequently, works reliably only in a narrow frequency range of the arc current. At $840 \mathrm{~Hz}$ the voltage waveform is mainly stationary and $\tau$ cannot be evaluated at all. At $8.4 \mathrm{kHz}$ the fluctuations in $P$ cause, as explained above, strongly scattered and rather low $\tau$ values. The $3.6 \mathrm{kHz}$ test current provided reasonable results for $\tau$ in the range $3 \mathrm{~S}<g<13 \mathrm{~S}$. At very low conductances, unexpected low $\tau$ values resulted and at very high values a strong scattering is observable. The evaluation method is very sensitive with respect to the chosen test current frequency. Evaluation of $\tau$ from experiments with $1 \mathrm{kHz}$ and $5.8 \mathrm{kHz}$ show comparably bad results as those from $840 \mathrm{~Hz}$ and $8.4 \mathrm{kHz}$, respectively.

\section{B. Staircase current method D}

1) Measurement results: Four identical experiments were performed with step wise increasing current. A typical current waveform is plotted in Fig. 3a). The current waveform features large slopes with $d i / d t>7 \mathrm{kA} / \mathrm{ms}$ to show deviations from the stationary curve. Each current step is followed by current plateau for $\Delta t=230 \mu \mathrm{s}$ of nearly constant current with slopes $d i / d t<0.07 \mathrm{kA} / \mathrm{ms}$. The current step is $\Delta I \approx 50 \ldots 100 \mathrm{~A}$.

The measured arc voltage is also plotted in Fig. 3a). For each current step, a voltage peak with an exponential decay of length $t_{\text {fast }}=10 \ldots 15 \mu \mathrm{s}$ is observable. A quasi stationary arc voltage section follows. This gives a first rough estimation of the thermal inertia $\tau$. During the constant current phase, the arc voltage is not fully constant but fluctuations with time constants of $t_{\text {slow }}>500 \mu \mathrm{s}$ occur. This behavior is due to fluctuations of the arc geometry or in its temperature distribution and is not described by the black-box eq. (4). Thus, even a perfect parameter evaluation method will show a certain error in predicting the quasi stationary behavior. However, the large difference in the dynamics $t_{\text {slow }} \gg t_{\text {fast }}$ justifies a $\tau$ extraction with a minimal error.

2) Parameter evaluation results: The resulting arc cooling power during one experiment with staircase like current is shown in Fig. 3b). The measurements $P_{m}$ are plotted with a solid line and the quasi-stationary values $P_{j}$ from the constant current phases are indicated with open circles. They were extracted as the mean value of the arc voltage during the constant current sections starting $50 \mu$ s after the current step 

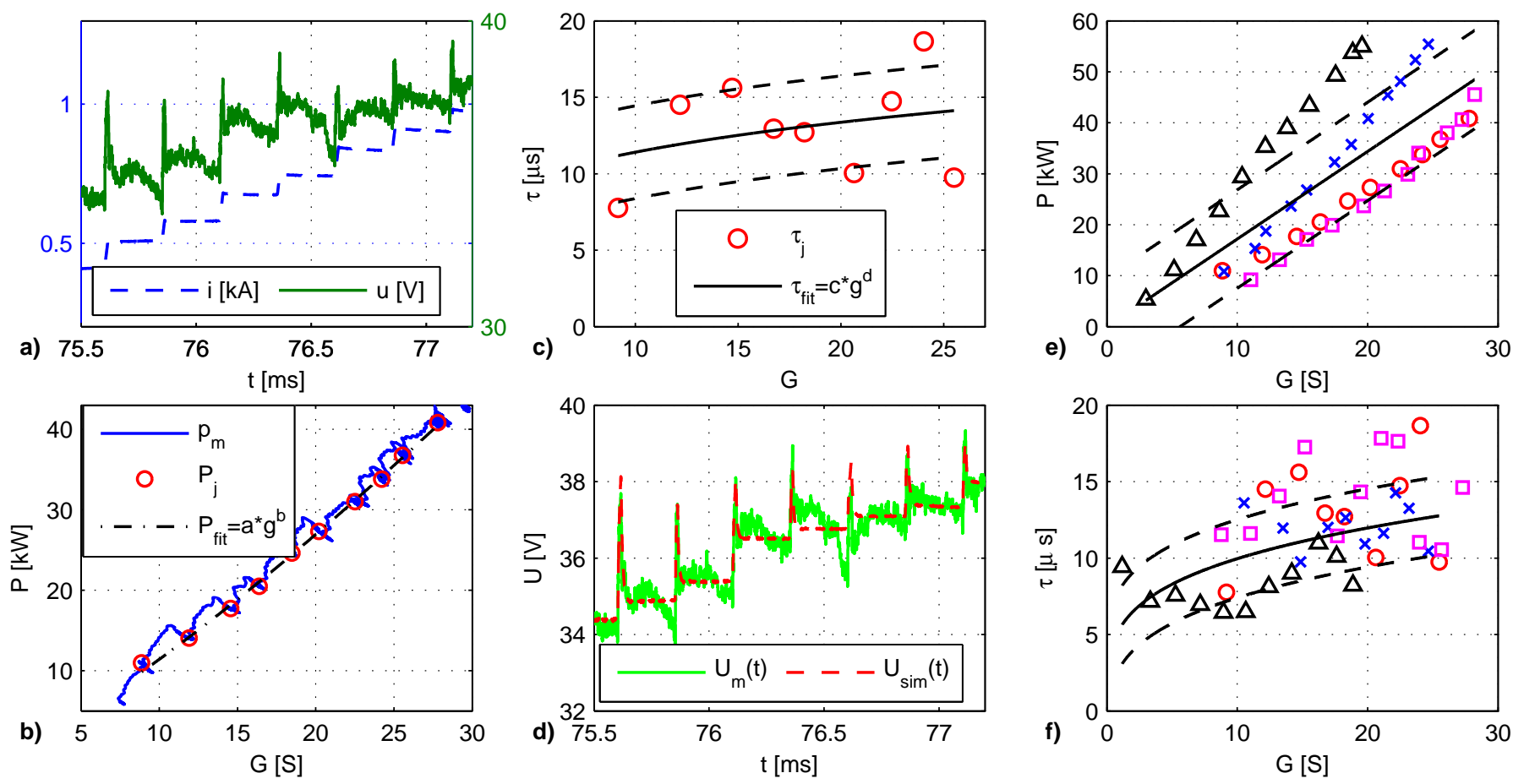

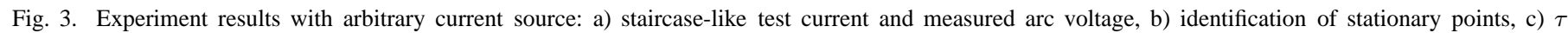
evaluated with method $\mathrm{D}, \mathrm{d}$ ) comparison of measured and recalculated voltage, e)-f) arc parameters extracted with method D from four identical experiments.

up to the next step. This assured that the fast dynamics have already vanished and the slow dynamics are averaged out. The stationary arc characteristic is approximately described by $P_{\text {fit }}=a \cdot\left(g / g_{0}\right)^{b}$ with $a=659 \mathrm{~W}, b=1.24$ and $g_{0}=1 \mathrm{~S}$. From the shape of the $P_{j}\left(G_{j}\right)$ curve (cf. Fig. 3b) ) it is possible to judge if a linearizing between the points is reasonable or if another measurement with smaller step size is required. Moreover, a large scatter in $P_{j}\left(G_{j}\right)$ would indicate that the external physical conditions are not maintained sufficiently constant.

The extracted $\tau(g)$ values are displayed in Fig. 3c). A general increase with increasing conductance can be seen and $\tau(g)$ can be approximately described by $\tau_{f i t}=c \cdot\left(g / g_{0}\right)^{d}$ with $c=6.7 \mu \mathrm{s}, d=0.23$ and $g_{0}=1 \mathrm{~S}$. The standard deviation is $\approx 4 \mu \mathrm{s}$.

3) Recalculation of step current experiment with evaluated arc parameters: For consistency check, the arc voltage can be recalculated with the black-box model eq. (4) using the previously evaluated discrete values of $\tau_{j}$ and linear interpolations between $P_{j}$. In Fig. 3d), the recalculated arc voltage waveform (dashed line) is compared with the measured one (solid line). The dynamic behavior corresponds correctly to the measured arc voltage. The stationary behavior does that only on average, as the slow stationary dynamics were neglected as described above. This holds true for the general fluctuations, but also for the sudden voltage drop at $76.6 \mathrm{~ms}$.

In Figs. 4e) and f), the evaluated parameters of four subsequent experiments with identical staircase-like currents are shown. In all experiments the dynamic arc behavior was similar, but the average arc voltages differed by approximately $5 \mathrm{~V}$. This can be seen in the $P(g)$ curves of Fig. 3e). While all $P(g)$ curves are nearly free of scattering in themselves, different $P(g)$ curves result from shot to shot, an indication of the different states of the arc in the different experiments. Interestingly, all four experiments result in similar $\tau(g)$, as shown in Fig. 3f). Consequently, $P$ seems to vary more from experiment to experiment than $\tau$.

4) Recalculation of $L C$-experiments with parameters evaluated from step-experiments: The parameters extracted by method D from the step current measurements were also used to recalculate the arc voltage of the experiments in the LCcircuit described above. Therefore, the parameter curves were analytically interpolated by the functions shown in Figs. 3e) and f) (solid lines).

$$
P(g)=a \cdot g \quad \text { and } \quad \tau(g)=c \cdot\left(g / g_{0}\right)^{d}
$$

with $a=1718 \mathrm{~W} / \mathrm{s}, c=5.4 \mu \mathrm{s}, d=0.26$, and $g_{0}=1 \mathrm{~s}$. Again eq. (4) was used to model the arc.

The recalculated voltage waveforms (dashed lines) are compared with the measured ones (solid lines) in Fig. 4. The recalculation result is very satisfying, deviations only occur directly after current-zero as the re-ignition is not covered in this black-box model.

\section{DISCUSSION}

An accurate measurement of dynamic arc current and voltage is very challenging, even with suitable measurement equipment. In addition, the arc may change its characteristics during a measurement due to elongation, arc root movement, as well as magnetically or thermally caused movement of the arc column. This results in continuous and stochastic arc voltage fluctuations. Hence, the quality of the determination 


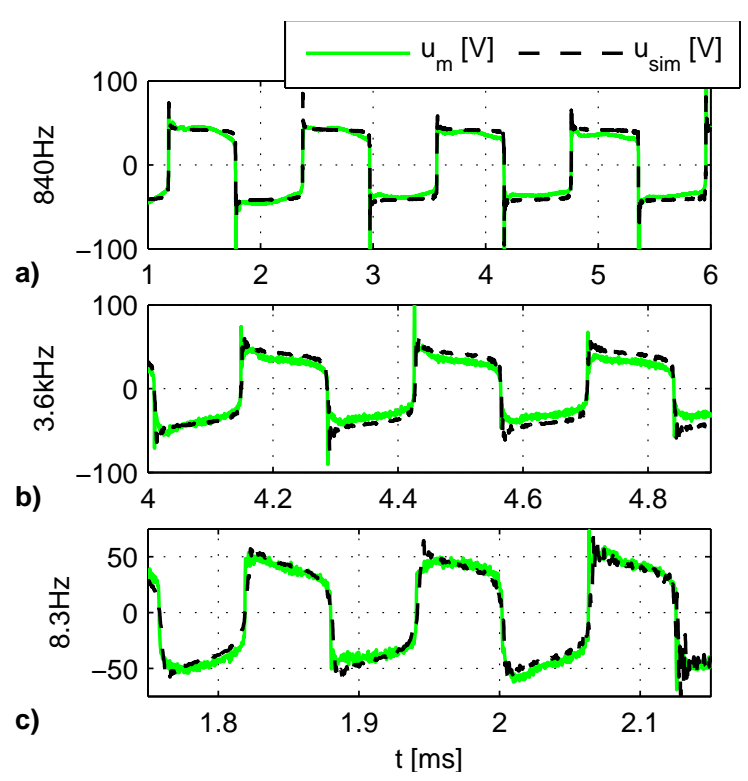

Fig. 4. Recalculation of the voltage waveforms for a sinusoidal current by the parameter functions extracted with the staircase-method

algorithms mainly depends on the ability to extract from a poor signal to noise ratio that part of the behavior that is governed by the chosen energy balance equation.

Method $A$ is very easy to implement, not sensitive to noise in the measurement signal, requires no specific current waveform, and often provides very acceptable results [14], [9]. However, the selected parameter functions are fitted to the whole experiment and thus typically to a wide range of conductances $g$. Even if it is possible to find parameter functions for $P$ and $\tau$ that are valid over the whole range of $g$, it is not obvious that the evaluated parameters are constant over the whole experiment. In our case, method $A$ was only successfull because it was fitted to all three measurements at different test current frequencies simultaneously. The best fit to the experiment at $f=840 \mathrm{~Hz}$ results in a poor recalculation of the arc voltage of the experiment at $f=8.3 \mathrm{kHz}$.

Method $B$ provides the benefit of independent $P$ and $\tau$ determination but requires a current waveform with sufficient current zero crossings and sufficient instances with $\dot{g}=0$. An oscillating current waveform with decreasing amplitude and decreasing current gradient is thus ideally suited. Alternatively, multiple measurements may be combined to collect sufficient extractable points. Handling numerical challenges is critical in the implementation of method B: Firstly, fluctuations in the measurement signal lead to instances with $\dot{g}=0$ that are part of the arc dynamics but are easily misinterpreted as stationary points. Secondly, at current zero the values $g$ and $\dot{g}$ are not defined, due to division by zero, and interpolation methods must be used. Thirdly, any time shift between voltage and current zero crossing leads to non reasonable $\tau$ values. Such a shift occurs quite regularly and can be the consequence of a measurement offset or a delay from the measurement equipment and long cables. The cooling power is successfully evaluated only for low frequencies as the arc is truly stationary at the current peak only for these current shapes. For increasing test currents frequency, the arc is no longer in the stationary state and this leads to an increased scattering in the evaluation. The opposite is true for evaluation of $\tau$. From stationary arcs (low frequency oscillations), the thermal time constant cannot be evaluated. To get reliable data from method $B$, at least two measurements with significantly different oscillation frequencies need to be performed.

Method $C$ strongly relies on the validity of eq. (4) because it determines $P$ and $\tau$ simultaneously from the dynamic waveform. Consequently, only a narrow band of current frequency allows an accurate parameter extraction. In our example, only the measurement at $3.6 \mathrm{kHz}$ was successful. We have performed experiments with slightly different test current frequency $(2.5 \mathrm{kHz}$ and $5.5 \mathrm{kHz}$; not shown here) and the evaluation showed considerable less accurate results, similar to the ones shown for $840 \mathrm{~Hz}$ and $8.3 \mathrm{kHz}$. Its graphical implementation does a certain intrinsical averaging through the straight line fitting but has strong error propagation for $\tau$ and is thus very vulnerable to arc fluctuations. Consequently, it requires large effort in implementation to deal with all the numerical problems and a manual identification of poorly extracted points.

Reliable evaluation of $P$ and $\tau$ with low scatter can be done using the new method $D$. From a single experiment, it is possible to determine the arc characteristic of the entire conductance range. From Fig. 4a) it can be seen that the arc voltage is not constant during the constant current phase. This corresponds to arc elongations, movements, or changes in the temperature distribution [24]. Thus only an average arc characteristic can be evaluated. However, from Fig. 4e) it can be seen that it is even possible to distinguish these different states of the arc from repetitive experiments. Of the four different experiments, three different cooling powers have been determined, which indicates three different arc lengths at the instant of the measurement. The lowest value, which corresponds to the shortest arc length, is found in two experiments. These differences in the state of the arc originate from the period before the staircase-like test current is applied, as a low current arc is burning for $100 \mathrm{~ms}$ before the experiment to minimize the effects of the ignition wire.

In addition to an improved accuracy in the arc parameter determination, method $\mathrm{D}$ is the only one that allows to judge if the chosen model equations are reasonable. From the exponential shape of arc voltage measurement in response to the current step of the presented experiments it can be confirmed that the behavior only depends on the deviation of the arc conductance from its stationary state and that it can be described with a simple time constant $\tau(g)$. The chosen power law relation for $P$ and $\tau$ with respect to $g$ holds true for the presented arc configuration. But, this may be different for other arcing conditions and always needs to be carefully checked. Of course, all the benefits described above come at the price of a substantially more challenging creation of the necessary specifically shaped current waveforms.

\section{CONCLUSiOn AND OUTLOOK}

Despite the recent advances in physical arc modeling, blackbox modeling is still a valuable tool to efficiently simulate 
arc-network interaction. This can be for example current interruption in power networks or the interaction between an arc chamber and a parallel LC-circuit in a passive resonance HVDC circuit breaker. The most important challenge in blackbox modeling is the choice of a parameter function to describe the arc and the determination of the parameter values for all states of the arc. In this contribution, the limitations of conventional parameter determination methods from experiments with sinusoidal test currents have been demonstrated on the example of a free burning arc. The problems are mainly threefold: a) the sensitivity of the classical methods to arc fluctuations, b) the limited spread of $\dot{I}$ in sinusoidal test currents and consequently the limited amount of information that can be extracted, and c) the necessity to make an a priori assumption of parameter function and model equation without the possibility of validation.

In this contribution an improved direct method to determine the arc characteristic by using more complex current waveforms is proposed. For the first time, the arc voltage was measured in response to a step current. It was demonstrated on the example of a free burning arc that it is possible to determine the arc characteristics with very high accuracy. It was even possible to distinguish different states of the arc in subsequent identical experiments. In addition, it was possible to confirm that commonly used arc model equations are suitable descriptions of the arc characteristics.

The ability of improved parameter extraction by using complex current waveforms is expected to become very helpful in future research on high current arcs. Other current waveforms besides the current steps may also be beneficial for more precise or more efficient arc parameter determination and will be investigated in the future.

\section{ACKNOWLEDGMENT}

The work is financially supported by ABB Switzerland Ltd, Corporate Research. The measurements with the LC-circuit were performed by Daniel Rothmund in the framework of a semester thesis.

\section{REFERENCES}

[1] J. L. Zhang, J. D. Yan, A. B. Murphy, W. Hall and M.T.C. Fang, Computational investigation of arc behavior in an auto-expansion circuit breaker contaminated by ablated nozzle vapor, IEEE Trans. Plasma Sci., No. 30(2), pp. 706-719, 2002.

[2] A. A. Iordanidis, C. M. Franck Self-consistent radiation-based simulation of electric arcs: II. Application to gas circuit breakers, J. Phys. D: Appl. Phys., Vol. 41(13), 135206, 2008.

[3] J. Sedlacek, Z. Vostracky, et.al. Optimization of high-voltage self-blast interrupters by gas flow and electric field computations, IEEE Trans. Power Del., Vol. 18(4), pp. 1228-1235, 2003.

[4] H. K. Kim, et.al., Approximation Model-Assisted Optimization Technique to Improve Capacitive Current Interrupting Performance of Gas Circuit Breaker, IEEE Trans. Magn., Vol. 45(3), pp. 1574-1577, 2009.

[5] T. Christen, M. Seeger, Current interruption limit and resistance on the self-similar electric arc, J. Phys. D: Appl. Phys, No. 97, 106108, 2005.

[6] R. Smeets, V. Kertsz, Evaluation of high-voltage circuit breaker performance with a validated arc model, IEE Proc.- Gener. Transm. Distrib., Vol. 147, No. 2, pp. 121-125, Mar. 2000.

[7] D. O. Bishop, A method of determining the dynamic characteristics of electric arcs, Proceedings of the IEE - Part IV: Institution Monographs, No. 101, pp. 18-26, 1954.

[8] M. Seeger, et.al., An integral arc model for ablation controlled arcs based on CFD simulations J. Phys. D: Appl. Phys., Vol. 39, No. 10, 2006
[9] H. Nakao, Y. Nakagoshi, M. Hatano and T. Koshizuka, D.C. Current Interruption in HVDC SF6 Gas MRTB by Means of Self-Excited Oscillation Superimposition, IEEE Trans. Power Del., Vol. 16, No. 4, Oct. 2001.

[10] C. M. Franck, HVDC Circuit Breakers: A Review Identifying Future Research Needs, IEEE. Trans. Power Del., Vol. 26, No. 2, Apr. 2011.

[11] C. Leu, Exitation and course of current chopping oscillation as an intermittent oscillation in SF6, $9^{\text {th }}$ International Conference on Switching Arc Phenomena (SAP 2001), Lodz, 2001.

[12] O. Mayr, Beiträge zur Theorie des statischen und des dynamischen Lichtbogens, Arch. f. El.-Tech., vd. 37, pp 588, Germany, Dec. 1943.

[13] A. N. Cassie, Arc rupture and circuit severity: a new theory, CIGREReport, bd. 102, pp 1-16, 1939.

[14] J. Schwarz, Berechnung von Schaltvorgängen mit einer zweifach modifizierten Mayr-Gleichung, Elektrotechnische Zeitschrift (ETZ-A), bd. 93, pp 386, Germany, 1972.

[15] A. Ahmethodzic, M. Kapetanovic, K. Sokolija and R. P. P. Smeets, Linking a Physical Arc Model with a Black Box Arc Model and Verification, IEEE Trans. Dielectr. Electr. Insul., Vol. 18, No. 4, Aug. 2011.

[16] D. Andersson, M. Barrault, D. Durfournet, M. Hrabosvsky, M. Kapetanovic et al., Applications of black box modeling to circuit breakers, Electra 149 (Aug.), p. 40-71, 1993.

[17] M. Kapetanovic, High Voltage Circuit Breakers, Book: ETF- Faculty of Electrical Engineering, Sarajevo, ISBN 978-9958-629-39-6,pp 71-76, 2011.

[18] G. Pietsch, H. Rijanto and G. Thiel, Schaltlichtbögen im elektrischen Netz, Elektrotechnische Zeitschrift (ETZ-A), vol. 96, pp. 222-226, Germany, 1975.

[19] Hendro Rijanto, Ein experimentelles Verfahren zur Bestimmung von Lichtbogenkenngroessen - Stromueberlagerungsverfahren, Ph.D. Thesis (german), Technical University Hannover, Hannover, Germany, 93 pages, Feb. 1975.

[20] R. Amsinck, Verfahren zur Ermittlung der das Ausschaltverhalten bestimmender Lichtbogenkenngrößen, Elektrotechnische Zeitschrift (ETZA), vol. 98, pp. 566, Germany, 1977.

[21] Rainer Ruppe, Experimentelle und theoretische Untersuchungen am axial bestroemten Wechselstromlichtbogen vor dem Stromnulldurchgang, Ph.D. Thesis (german), Technical University Ilmenau, Ilmenau, Germany, 101 pages, Mar. 1980.

[22] H. Drebenstedt, W. Rother, C. Weber and P. Zahlmann, Ein verbessertes Verfahren zur Bestimmung der charakteristischen Funktionen des Zweipolmodells für Schaltlichtbögen, 28. Internat. Wiss. Kolloquium, TH Ilmenau, 1983.

[23] M. M. Walter, C. M. Franck Flexible pulsed DC-source for investigation of HVDC circuit breaker arc resistance, Int. Conf. on Gas Discharges and their Applications, Greifswald Germany, pp. 170, 2010.

[24] A.D. Stokes, W.T. Oppenlander, Electric arcs in open air, J. Phys. D: Appl. Phys., Vol 24, Jan. 1991.

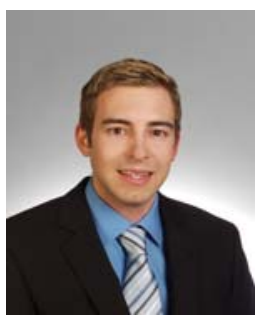

Michael M. Walter was born in Zurich in Switzerland in 1984. He received his Master Degree in Electrical Engineering and Information Technology from ETH- Zurich in 2010. Currently he is researching on Switching arcs in passive resonant HVDC circuit breakers in the scope of his Ph.D. at ETH- Zurich. His employees for industrial projects and internships during his Master-Studies include the Mitsubishi Electric Corporation in Amagasaki city, Japan, the ABB Switzerland AG (Corporate Research in Dättwil and the ATPT department in Turgi) and the Institute Mihajlo Pupin in Belgrade, Serbia. 


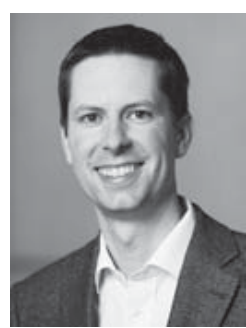

Christian M. Franck (M'04-SM'11) received a diploma in physics from the University of Kiel, Germany in 1999 and the Ph.D. degree in physics from the University of Greifswald, Greifswald Germany in 2003. He was with the Swiss corporate research center of ABB from 2003-2009 as a Scientist and Group Leader for gas circuit breakers and high-voltage systems. Currently, he is an Assistant Professor for High Voltage Technology at the Swiss Federal Institute of Technology (ETH), Zurich, Switzerland. 